\title{
Dosimétrie biologique en cas d'exposition radiologique d'un grand nombre d'individus : apport de la détection automatique des dicentriques
}

\author{
A. VAURIJOUX ${ }^{1}$, G. GRUEL ${ }^{1}$, S. ROCH-LEFÈVRE ${ }^{1}$, E. GRÉGOIRE ${ }^{1}$, \\ C. MARTIN ${ }^{1}$, P. VOISIN ${ }^{1}$, PH. VOISIN ${ }^{1}$, L. ROY $^{1}$
}

(Manuscrit reçu le 12 novembre 2009, accepté le 27 février 2010)

RÉSUMÉ En cas d'accident radiologique impactant un grand nombre d'individus, il est primordial de différencier rapidement les individus exposés des individus non exposés. Le laboratoire de dosimétrie biologique de l'IRSN développe des méthodes pour répondre à ce type de situation. La méthode de référence est basée sur une estimation rapide de la dose après comptage des dicentriques et des anneaux dans 50 métaphases. À l'issue de cette étape de tri de la population exposée, une estimation plus précise de la dose reçue peut être nécessaire. Cette étape est longue car elle nécessite l'observation des aberrations chromosomiques dans au moins 500 métaphases. Pour améliorer cette méthodologie en deux étapes, nous avons étudié l'utilisation d'un logiciel de détection automatique des dicentriques dans un cas réel d'accident radiologique impliquant 63 victimes. Cette méthode a été comparée aux méthodes manuelles de références. La comparaison des doses obtenues montre que la méthode automatique peut remplacer les deux étapes du comptage manuel (50 et 500 métaphases), car elle cumule les avantages de chacune d'elles : rapidité et précision.

ABSTRACT Biological dosimetry in the case of large-scale accidental overexposure to ionising radiation: contribution of the automatic detection of dicentrics.

Following large-scale accidental overexposure to ionising radiation, a rapid triage of the exposed population can be performed by scoring dicentrics and ring chromosomes in 50 metaphases. This is rapid but not accurate. Following this triage step, the dose can be estimated by scoring 500 metaphases. This is lengthy but very accurate. To improve the methodology, we propose using software for automatic dicentric scoring, which was tested on 63 victims of a radiological accident. The method using automatic dicentric scoring was compared with the standard methods of manual scoring. The comparison of the doses obtained shows that the automatic method could replace the two steps of the manual scoring (50 and 500 metaphases). In fact, the automatic scoring method is more accurate than the manual scoring of 50 metaphases and can therefore be used for triage.

Keywords: Biological dosimetry / serious accidental overexposure / triage / manual scoring / automatic dicentric detection

\footnotetext{
1 Laboratoire de Dosimétrie Biologique, IRSN, BP 17, 92262 Fontenay-aux-Roses, France.
} 


\section{Introduction}

Suite à un accident radiologique conduisant à une exposition aux rayonnements ionisants, il est essentiel d'estimer la dose reçue par les victimes. Cette estimation peut s'appuyer sur la dosimétrie physique et/ou sur la dosimétrie biologique et intervient après un diagnostic clinique réalisé par un médecin. Cependant, une reconstitution précise du scénario d'irradiation est généralement difficile à effectuer d'emblée. Dans ce cas, seule la dosimétrie biologique permet de confirmer le diagnostic clinique d'exposition. Elle est alors une aide indispensable à une prise en charge médicale adaptée des victimes.

Le laboratoire de dosimétrie biologique de l'IRSN réalise depuis 17 ans des expertises dosimétriques suite à des suspicions d'accidents radiologiques. La technique de référence utilisée par le laboratoire est basée sur le dénombrement des aberrations chromosomiques instables (dicentriques, anneaux centriques et fragments acentriques) observées sur des lymphocytes du sang circulant. Il est effectué sur 500 métaphases, après coloration des chromosomes au Giemsa (Roy et al., 1996).

Dans le cas particulier d'un accident d'exposition aux rayonnements ionisants impliquant un grand nombre de personnes, un recensement et une classification des victimes basés sur les symptômes cliniques sont faits en première intention. Pour confirmer ce diagnostic médical, une dosimétrie biologique de crise est réalisée pour chacune des victimes. Elle consiste à appliquer la méthode de référence décrite ci-dessus en « mode dégradé » puisque seulement 50 métaphases seront analysées par individu. Elle permet d'obtenir rapidement une première estimation des niveaux d'exposition, mais la dose ainsi estimée est peu précise. Cette dosimétrie biologique de crise est donc suivie d'une estimation plus précise de la dose basée sur l'observation des 500 métaphases. L'ensemble de cette procédure d'expertise est longue. Pour augmenter la rapidité d'analyse sans diminuer le nombre de métaphases comptées (gage de la précision des estimations de doses), l'utilisation de la détection automatique des dicentriques a donc été proposée.

Dans cette étude, nous allons analyser l'intérêt de l'utilisation de la détection automatique des dicentriques, par un logiciel commercial, en cas d'accident d'exposition aux rayonnements ionisants impliquant un grand nombre d'individus. Nous avons pris comme modèle un accident survenu en 2006 à Dakar (Sénégal) impliquant 63 individus potentiellement exposés à une source d'iridium-192 et pour lequel le laboratoire de dosimétrie biologique de l'IRSN a été chargé de la gestion et de la réalisation des expertises en dosimétrie biologique. 


\section{2. État de l'art de la dosimétrie biologique}

La technique de référence basée sur le dénombrement des aberrations instables (dicentriques, anneaux centriques et fragments acentriques) peut être appliquée lors d'un accident radiologique de moins de 6 mois (Grégoire et al., 2010). Cette limite est due au caractère instable des aberrations analysées. Elle a un seuil de sensibilité de 0,16 Gy avec comme borne basse et haute de l'intervalle de confiance 0,01 Gy et 0,41 Gy respectivement. En routine, l'analyse manuelle de 500 métaphases est faite en 10 heures par un opérateur formé et entrainé (Roy et al., 2007). Cette méthode est donc applicable sur un nombre d'individus inférieur à 10 . Le temps d'analyse devient problématique lors d'un accident impliquant un grand nombre d'individus.

Dans ce dernier cas, un tri rapide des victimes est réalisé avec la technique de référence en «mode dégradé » (50 métaphases analysées). Le seuil de sensibilité n'est alors que de 0,53 Gy avec comme borne basse et haute de l'intervalle de confiance 0,002 Gy et 1,47 Gy respectivement. Cette procédure a été appliquée lors d'un accident de surexposition radiologique qui a eu lieu en Géorgie en 1997 (Voisin et al., 2001). Le tri de 85 victimes potentielles a été réalisé par l'analyse sur 50 métaphases. Avec cette méthode, les doses ont été sous-estimées pour $82 \%$ des individus exposés à une dose inférieure à $1 \mathrm{~Gy}$. Ces résultats étant peu satisfaisants des améliorations ont été expérimentées notamment avec l'automatisation de la détection des dicentriques. En effet, dans la littérature, deux laboratoires ont développés leur système de détection automatique des dicentriques (Finnon et Lloyd, 1992 ; Bayley et al., 1991). Ils ont obtenus un seuil de sensibilité de 0,25 Gy sur un modèle d'irradiation in vitro (Bayley et al., 1991). Ces résultats étant prometteurs, nous avons appliqué la détection automatique des dicentriques à cette situation accidentelle particulière dans l'objectif d'améliorer : (i) le temps d'analyse qui doit être le plus court possible et (ii) le seuil de sensibilité de la dose qui doit être le plus proche possible de celui de la technique de référence.

\section{Matériel et méthode}

\subsection{Contexte de l'accident de Dakar}

De juin à août 2006, des travailleurs utilisant des appareils de gammagraphie ont été exposés à une source d'iridium-192 à Dakar (Sénégal) puis à Abidjan (Côte d'Ivoire). Ces appareils de gammagraphie sont constitués d'un container de stockage, d'un tube d'éjection et d'une source maintenue par un câble permettant la manipulation de cette dernière à distance et donc sans risque pour l'opérateur. La reconstitution du scénario de l'accident a permis de mettre en évidence que la 
source s'était détachée du câble puis était restée bloquée dans le tube d'éjection lorsque l'opérateur avait cru la faire rentrer dans le container de stockage (Clairand et al., 2008). La source d'iridium est restée dans la gaine d'éjection plusieurs semaines. Cette gaine d'éjection, initialement localisée à Dakar (Sénégal), a été envoyée à Abidjan où la source a finalement été découverte. À Dakar comme à Abidjan de nombreuses personnes ont potentiellement été exposées à cette source non protégée et fortement irradiante.

Suite à la découverte de la source, un tri (50 métaphases analysées) suivi d'une estimation plus précise de la dose (250/500 métaphases analysées) ont été effectués pour 63 individus identifiés comme ayant potentiellement pu être exposés aux rayonnements de la source.

\subsection{Collecte des échantillons}

Trois mois après le début de l'incident, les victimes potentielles ont bénéficié d'un examen médical durant laquelle elles ont rempli un questionnaire précis sur leur état de santé et leur localisation par rapport à la source. Un prélèvement de sang a été réalisé sur anticoagulant héparine/lithium après avoir obtenu le consentement éclairé de chaque individu.

Les échantillons ont été envoyés au laboratoire en trois fois : deux envois de Dakar et un envoi d'Abidjan. À la réception, les échantillons ont été numérotés pour respecter l'anonymat des individus.

\subsection{Culture de lymphocytes et préparation des chromosomes}

Pour chaque échantillon de sang, la division des lymphocytes a été induite en ajoutant $0,5 \mathrm{ml}$ de sang à $5 \mathrm{ml}$ de milieu RPMI 1640 supplémenté, avec un temps de culture de 48 heures ou de 50 heures à $37^{\circ} \mathrm{C}$ et un ajout de la colcémide à 46 heures (inhibiteur mitotique). Les métaphases ont ensuite été étalées sur lame après un choc hypotonique et des fixations. Ces lames ont été colorées par la technique de Fluorescence Plus Giemsa (FPG) (Roy et al., 1996).

\subsection{Recherche et acquisition des métaphases}

Les métaphases obtenues ont été analysées avec un microscope Axioplan 2 Imaging (Zeiss Oberkochen, Germany) équipé d'une caméra (Jai ; Copenhagen, Denmark) et d'une platine motorisée (Märzhäuser ; Wetzlar, Germany). Les positions des métaphases ont été identifiées automatiquement par le logiciel Metafer 4 (version 3.5.101; MetaSystems Gmbh ; Baden-Württemberg, Germany) avec l'objectif à grossissement $\times 10$ (Zeiss ; Oberkochen, Germany). Ensuite les 

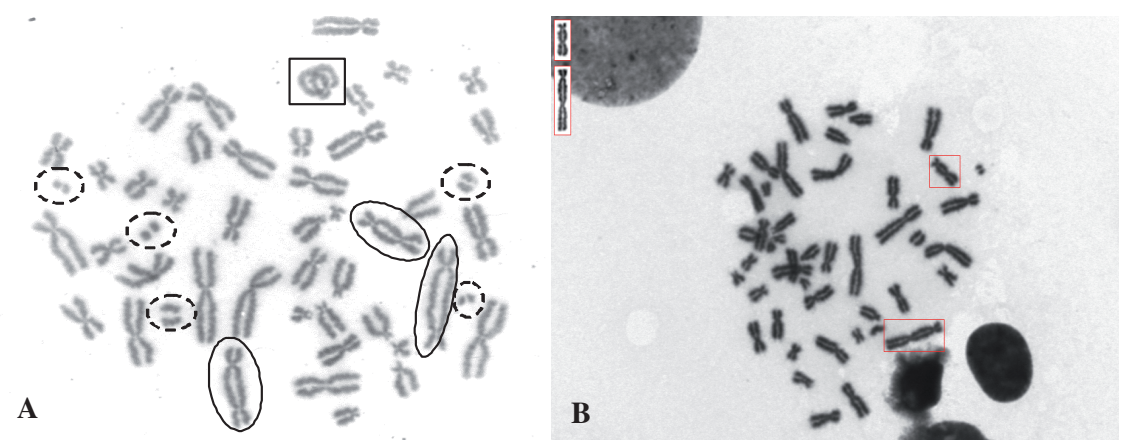

Figure 1 - Images de métaphases acquises au grossissement $\times 63$ par le logiciel Metafer 4 (MetaSystems Gmbh). (A) Comptage manuel : les dicentriques (cercles), les anneaux (carré) et les fragments (cercles pointillés) sont dénombrés. (B) Détection automatique : le logiciel DCScore détecte les objets et encadre ceux qu'il détecte comme étant des dicentriques (carrés).

Metaphases pictures acquired with the $\times 63$ objective by Metafer 4 software (MetaSystems Gmbh). (A) Manual scoring: dicentrics (circles), centric rings (square) and fragments (dotted circles) were scored. (B) Automatic detection: DCScore software detect the objects and select the objects that it detect like been dicentrics (squares).

métaphases ont été acquises avec l'objectif à grossissement $\times 63$ (Zeiss ; Oberkochen, Germany). Les images des métaphases ont été utilisées à la fois pour l'analyse manuelle et pour l'analyse automatique.

\subsection{Analyse des métaphases par la méthode manuelle}

À partir des métaphases acquises au grossissement $\times 63$, seules les métaphases complètes (46 centromères) ont été analysées. Sur chacune d'elles, les dicentriques, les anneaux centriques et les fragments acentriques observés ont été dénombrés (Fig. 1A). Les résultats ont été exprimés en fréquence de dicentriques et d'anneaux par cellule. Afin d'identifier les individus les plus exposés, un tri sur 50 métaphases complètes (50 MS) a été effectué dans un premier temps. Dans un second temps, 250 à 500 métaphases complètes (250/500 MS) ont été analysées afin d'obtenir une meilleur sensibilité de la dose.

\subsection{Tri et estimation de la dose}

À l'issue de l'étape de tri (50 MS), les individus ont été répartis en 3 catégories suivant leur niveau d'exposition : la classe 0 où aucune dose n'est détectée ; la classe 1 où une dose positive est mesurée avec un intervalle de confiance à $95 \%$ incluant 0 Gy (différence non significative de 0 Gy) et la classe 2 où une dose positive est mesurée avec un intervalle de confiance à $95 \%$ n'incluant pas 0 Gy 
(différence significative de 0 Gy). La classe 1 correspond à des individus dont la fréquence de dicentriques est supérieure au bruit de fond de la population sans que la dose estimée correspondante soit significative (limite de la technique). C'est une classe intermédiaire capitale lors d'un tri car il y a une probabilité non nulle que les individus de cette classe passent dans la classe 2 lors de l'estimation de la dose finale avec 500 métaphases.

À partir de cette classification, les comptages ont été poursuivis en commençant prioritairement par la classe 2 puis la classe 1 et enfin la classe 0 . La dose finale a été déterminée en comptant d'abord 250 métaphases. La détection d'au moins un dicentrique a entrainé l'étendue du comptage à 500 métaphases (250/500 MS).

\subsection{Analyse expérimentale par détection automatique des dicentriques}

La détection automatique des dicentriques (ADS) a été effectuée sur 46 des 63 individus. Ces derniers ont été choisis en fonction de leur classification : tous les individus de la classe 1 et de la classe 2 ont été analysés, ainsi que 12 individus de la classe 0 , choisis au hasard.

La détection automatique des dicentriques (ADS) a été faite avec le logiciel DCScore (version 1.81 ; MetaSystems Gmbh ; Baden-Württemberg, Germany) sur les métaphases acquises au grossissement $\times 63$. Ce logiciel permet d'identifier comme chromosome et comme dicentrique tout objet correspondant à des critères de forme et de taille prédéfinis (Fig. 1B) (Schunck et al., 2004). Les objets proposés par le logiciel comme étant des dicentriques ont été passés en revue par un opérateur qui les a validés ou les a rejetés. Les résultats ont été exprimés en fréquence de dicentrique par cellule.

\subsection{Statistiques}

Pour les trois méthodes, les doses reçues ont été estimées en reportant les fréquences de dicentriques sur les courbes dose-effet respectivement établies à l'aide du comptage manuel ou du comptage automatique. Un intervalle de confiance à $95 \%$ a été calculé pour chaque estimation de dose (Fig. 2). Ces intervalles de confiance ont été estimés en reportant les bornes basses et hautes des intervalles de confiance des fréquences de dicentriques sur des courbes de déviation standard respectivement supérieure et inférieure (Papworth, 1975). Les intervalles de confiance des fréquences de dicentriques sont calculés à partir de la loi de Poisson. En effet, la distribution du nombre de dicentriques au sein des métaphases observées suit une loi de Poisson (Edwards et al., 1979). 


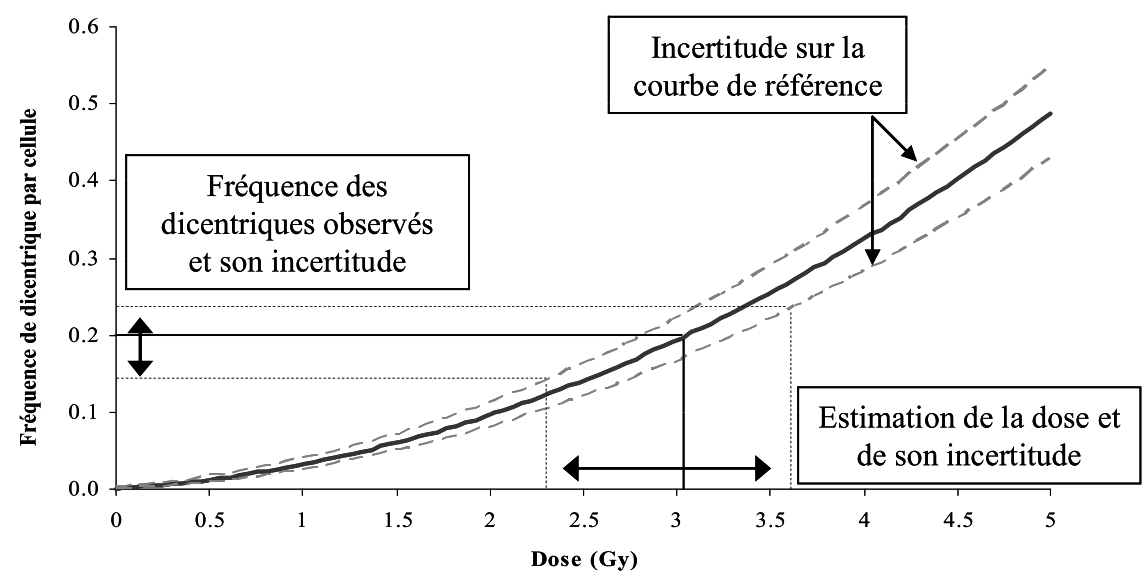

Figure 2 - Courbe dose-effet obtenue par la méthode ADS (ligne noire). Les courbes haute et basse représentent les courbes de déviation standard (lignes pointillés grises). La dose ainsi que son incertitude sont calculées par report de la fréquence de dicentriques observée et de son incertitude sur les courbes.

Dose-effect curves for the ADS method (bold black line). The upper and lower (grey dotted line) curves represent the standard deviations. The dose and its incertitude are calculated by correspondence of the dicentric yield observed and its incertitude on the curves.

Afin de comparer de manière globale les doses obtenues par les méthodes 250/500 MS et ADS, un test non-paramétrique de Wilcoxon a été appliqué. L'hypothèse nulle testée est la suivante : $\mathrm{H}_{0}: \mathrm{ADS}=500 \mathrm{MS}$ par rapport à une hypothèse alternative $\mathrm{H}_{1}: \mathrm{ADS} \neq 500 \mathrm{MS}$. La différence entre les méthodes 250/500 MS et ADS a été considérée comme significative lorsqu'à l'issue du test, la valeur absolue de la statistique $\mathrm{Z}$ issue du test était supérieure à 1,96 (p-valeur $<0,05)$.

\section{Résultats}

Les méthodes de comptage ont été comparées comme suit: 50 MS versus 250/500 MS et ADS versus 250/500 MS. Les points de comparaison ont été les temps d'analyse, la classification des doses obtenues suite a la phase de tri, l'estimation plus précise des doses et enfin le seuil de sensibilité de la dose.

\subsection{Temps de traitement par les différentes méthodes}

Le temps nécessaire pour l'analyse manuelle a été comparé à celui de l'analyse automatique des dicentriques. Les paramètres de temps analysés sont : (i) le temps de culture des lymphocytes, (ii) la préparation des lames, (iii) les temps de 


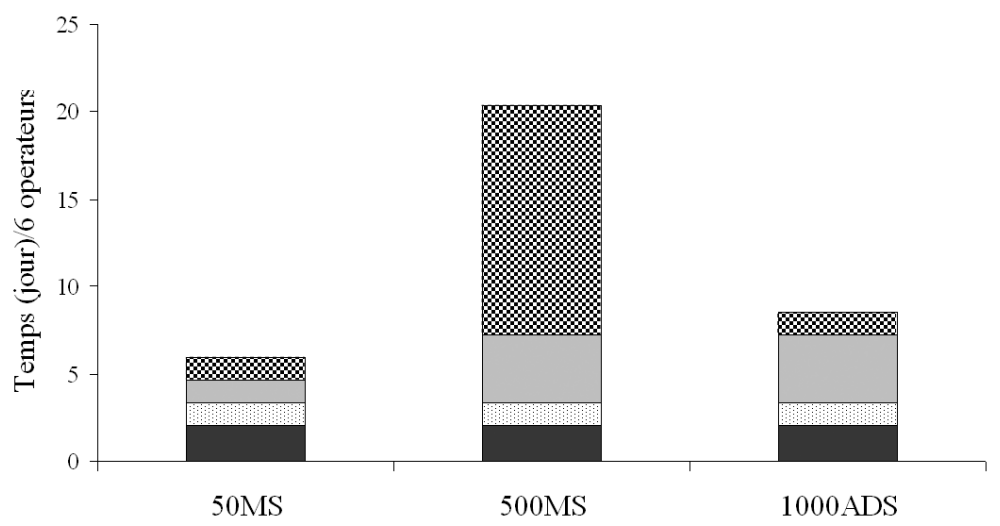

Figure 3-Temps passés pour chaque étape du protocole de dosimétrie biologique pour chaque méthode (50 MS, $500 \mathrm{MS}, 1000 \mathrm{ADS})$ : le temps de culture (noir), le temps de préparation des lames (points noirs), le temps de recherche et d'acquisition par le logiciel Metafer 4 (gris) et le temps d'analyse des métaphases (damier). Le temps est indiqué en jours de travail (équivalent à 8 h) pour 6 opérateurs mobilisés.

Total time needed to perform each step of biological dosimetry protocol for each method (50 MS, $500 \mathrm{MS}, 1000 \mathrm{ADS}$ ): the culture time (black), the time of slides preparation (black points), the time of metaphase searching and acquisition by the Metafer 4 software (grey) and the time of metaphases analysis (draughtboard). The time is shown in working days (8 hours) for 6 operators.

recherche et d'acquisition des métaphases par le logiciel Metafer 4 (MetaSystems Gmbh) et (iv) le temps d'analyse des métaphases dépendant du nombre de métaphases analysées mais également de la technique mis en œuvre (Fig. 3). Il faut noter que le temps d'analyse nécessaire pour les 63 victimes avec la méthode de détection automatique des dicentriques a été extrapolé à partir des résultats obtenus pour 46 d'entre eux.

Les résultats montrent que la méthode $50 \mathrm{MS}$ reste la plus rapide (5,9 jours) en comparaison à la méthode $\operatorname{ADS}(8,6$ jours). En revanche, la méthode 250/500 MS est beaucoup plus longue (20,4 jours).

\subsection{Classification des victimes}

Une classification des victimes en 3 groupes a été effectuée en première intention (voir «Matériels et méthodes »). Pour étudier les différences entre les méthodes $50 \mathrm{MS}$ et ADS et déterminer la plus efficace, les classifications obtenues avec ces 2 méthodes ont été comparés avec celle obtenue par la méthode 250/500 MS (méthode de référence). La répartition de chaque victime dans les 3 classes $(0,1$ ou 2) est donnée dans la figure 4. 


\section{TABLEAU I}

Doses obtenues pour chacune des 46 victimes de l'accident de surexposition aux rayonnements ionisants de Dakar. Les doses sont données avec leur intervalle de confiance à $95 \%$ pour les trois méthodes : $50 \mathrm{MS}, \mathbf{2 5 0 / 5 0 0}$ MS et ADS. Les individus sont classés en trois catégories : la classe 0 dans laquelle aucune dose n'est détectée ; la classe 1 dans laquelle une dose positive est détectée mais dont l'intervalle de confiance inférieur inclut la dose $0 \mathrm{~Gy}$ et la classe 2 dans laquelle une dose significativement positive est détectée. IC- : Borne inférieure de l'intervalle de confiance : IC+ : Borne supérieure de l'intervalle de confiance.

Doses obtained for 46 victims of radiological accident of Dakar. The doses and theirs $95 \%$ confidence intervals are indicated for the three methods: $50 \mathrm{MS}, 250 / 500 \mathrm{MS}$ and ADS. The individuals are classified in three categories: class 0 : no dose detected; class 1: a positive dose was measured with the lower confidence interval including $0 \mathrm{~Gy}$; and class 2: a significant positive dose is measured with the lower confidence interval above 0 Gy. IC-: lower confidence interval; IC+: upper confidence interval.

\begin{tabular}{|c|c|c|c|c|c|c|c|c|c|c|c|c|}
\hline \multirow{2}{*}{ Patient } & \multicolumn{4}{|c|}{ Méthode 250/500MS } & \multicolumn{4}{|c|}{ Méthode 50MS } & \multicolumn{4}{|c|}{ Méthode ADS } \\
\hline & $\begin{array}{l}\text { Dose } \\
\text { (Gy) }\end{array}$ & & IC + & Classe & $\begin{array}{l}\text { Dose } \\
\text { (Gy) }\end{array}$ & IC- & $\mathrm{IC}+$ & Classe & $\begin{array}{l}\text { Dose } \\
\text { (Gy) }\end{array}$ & & $\mathrm{IC}+$ & Classe \\
\hline 6 & 0 & - & - & \multirow{12}{*}{ 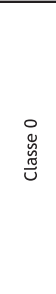 } & 0 & - & - & 0 & 0 & - & - & 0 \\
\hline 8 & 0 & - & - & & 0 & - & - & 0 & 0 & - & - & 0 \\
\hline 11 & 0 & - & - & & 0 & - & - & 0 & 0 & - & - & 0 \\
\hline 12 & 0 & - & - & & 0 & - & - & 0 & 0 & - & - & 0 \\
\hline 15 & 0 & - & - & & 0 & - & - & 0 & 0 & - & - & 0 \\
\hline 18 & 0 & - & - & & 0 & - & - & 0 & 0 & - & - & 0 \\
\hline 19 & 0 & - & - & & 0 & - & - & 0 & 0 & - & - & 0 \\
\hline 28 & 0 & - & - & & 0 & - & - & 0 & 0 & - & - & 0 \\
\hline 29 & 0 & - & - & & 0 & - & - & 0 & 0 & - & - & 0 \\
\hline 32 & 0 & - & - & & 0 & - & - & 0 & 0 & - & - & 0 \\
\hline 41 & 0 & - & - & & 0 & - & - & 0 & 0 & - & - & 0 \\
\hline 44 & 0 & - & - & & 0 & - & - & 0 & 0 & - & - & 0 \\
\hline 5 & 0.01 & 0 & 0.22 & \multirow{25}{*}{ 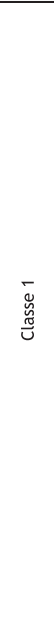 } & 0 & - & - & 0 & 0.05 & 0 & 0.46 & 1 \\
\hline 10 & 0.01 & 0 & 0.22 & & 0 & - & - & 0 & 0.12 & 0 & 0.47 & 1 \\
\hline 14 & 0.01 & 0 & 0.22 & & 0 & - & - & 0 & 0.09 & 0 & 0.53 & 1 \\
\hline 17 & 0.01 & 0 & 0.22 & & 0.3 & 0 & 1.24 & 1 & 0.04 & 0 & 0.42 & 1 \\
\hline 24 & 0.01 & 0 & 0.22 & & 0 & - & - & 0 & 0.03 & 0 & 0.41 & 1 \\
\hline 25 & 0.01 & 0 & 0.22 & & 0 & - & - & 0 & 0.066 & 0 & 0.47 & 1 \\
\hline 26 & 0.01 & 0 & 0.22 & & 0 & - & - & 0 & 0.07 & 0 & 0.49 & 1 \\
\hline 36 & 0.01 & 0 & 0.19 & & 0 & - & - & 0 & 0.07 & 0 & 0.49 & 1 \\
\hline 42 & 0.01 & 0 & 0.21 & & 0 & - & - & 0 & 0.17 & 0 & 0.56 & 1 \\
\hline 9 & 0.02 & 0 & 0.22 & & 0 & - & - & 0 & 0.21 & 0 & 0.63 & 1 \\
\hline 13 & 0.05 & 0 & 0.25 & & 0 & - & - & 0 & 0.05 & 0 & 0.44 & 1 \\
\hline 16 & 0.05 & 0 & 0.28 & & 0 & - & - & 0 & 0.05 & 0 & 0.44 & 1 \\
\hline 20 & 0.05 & 0 & 0.27 & & 0.3 & 0 & 1.24 & 1 & 0.08 & 0 & 0.53 & 1 \\
\hline 27 & 0.05 & 0 & 0.28 & & 0 & - & - & 0 & 0.30 & 0 & 0.67 & 1 \\
\hline 30 & 0.05 & 0 & 0.28 & & 0 & - & - & 0 & 0 & - & - & 0 \\
\hline 31 & 0.05 & 0 & 0.28 & & 0.16 & 0 & 0.8 & 1 & 0.183 & 0 & 0.58 & 1 \\
\hline 37 & 0.05 & 0 & 0.27 & & 0.3 & 0 & 1.24 & 1 & 0.08 & 0 & 0.51 & 1 \\
\hline 35 & 0.08 & 0 & 0.3 & & 0 & - & - & 0 & 0.18 & 0 & 0.51 & 1 \\
\hline 7 & 0.09 & 0 & 0.33 & & 0 & - & - & 0 & 0.07 & 0 & 0.49 & 1 \\
\hline 23 & 0.09 & 0 & 0.32 & & 0 & - & - & 0 & 0.22 & 0 & 0.58 & 1 \\
\hline 33 & 0.09 & 0 & 0.34 & & 0 & - & - & 0 & 0.07 & 0 & 0.49 & 1 \\
\hline 39 & 0.12 & 0 & 0.25 & & 0.3 & 0 & 1.24 & 1 & 0.14 & 0 & 0.50 & 1 \\
\hline 40 & 0.12 & 0 & 0.25 & & 0 & - & - & 0 & 0.14 & 0 & 0.50 & 1 \\
\hline 45 & 0.12 & 0 & 0.25 & & 0 & - & - & 0 & 0.03 & 0 & 0.40 & 1 \\
\hline 34 & 0.12 & 0 & 0.3 & & 0.3 & 0 & 1.24 & 1 & 0.23 & 0 & 0.56 & 1 \\
\hline 2 & 0.15 & 0.01 & 0.41 & \multirow{9}{*}{$\begin{array}{l}\stackrel{\Xi}{~} \\
\underset{\tilde{U}}{U}\end{array}$} & 0.53 & 0.002 & 1.47 & 2 & 0.39 & 0.07 & 0.77 & 2 \\
\hline 3 & 0.22 & 0.05 & 0.49 & & 0 & - & - & 0 & 0.44 & 0.12 & 0.81 & 2 \\
\hline 38 & 0.22 & 0.03 & 0.54 & & 0.3 & 0 & 1.24 & 1 & 0.22 & 0 & 0.58 & 1 \\
\hline 43 & 0.25 & 0.04 & 0.61 & & 0.53 & 0.02 & 1.47 & 2 & 0.34 & 0.01 & 0.72 & 2 \\
\hline 21 & 0.35 & 0.09 & 0.75 & & 0.53 & 0.02 & 1.47 & 2 & 0.65 & 0.32 & 1.03 & 2 \\
\hline 22 & 0.37 & 0.08 & 0.83 & & 0.3 & 0 & 1.24 & 1 & 0.63 & 0.31 & 1.01 & 2 \\
\hline 46 & 0.37 & 0.1 & 0.8 & & 0 & - & - & 0 & 0.73 & 0.37 & 1.15 & 2 \\
\hline 4 & 0.92 & 0.63 & 1.3 & & 1.03 & 0.34 & 2.00 & 2 & 0.86 & 0.56 & 1.21 & 2 \\
\hline 1 & 2.71 & 2.19 & 3.38 & & 2.39 & 1.57 & 3.44 & 2 & 2.28 & 1.90 & 2.70 & 2 \\
\hline
\end{tabular}




\section{0/500MS}

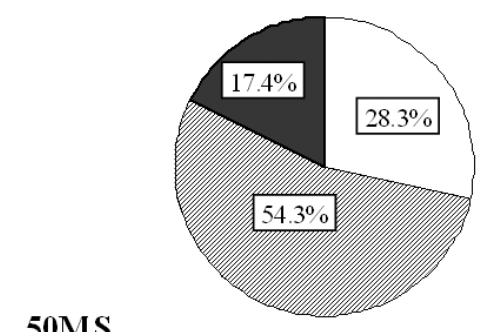

$50 \mathrm{MS}$

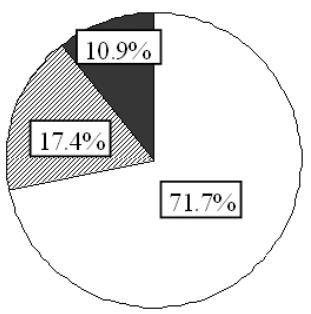

ADS

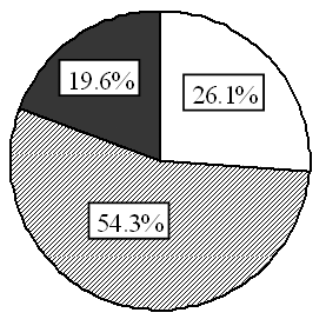

Figure 4 - Classification (en pourcentage) des victimes de l'accident d'exposition aux rayonnements ionisants de Dakar en fonction des niveaux d'exposition. Un diagramme circulaire est représenté pour chaque méthode : $50 \mathrm{MS}, 250 / 500 \mathrm{MS}$ et ADS. Les individus sont classés en trois catégories : la classe 0 dans laquelle aucune dose n'est détectée (blanc); la classe 1 dans laquelle une dose positive est détectée mais dont l'intervalle de confiance inférieur inclut la dose 0 Gy (hachuré) et la classe 2 dans laquelle une dose significativement positive est détectée (noir).

Dose classification obtained for victims of the radiological accident in Dakar. A pie chart is presented for each method: $50 \mathrm{MS}, 250 / 500 \mathrm{MS}$ and ADS. For each chart, the individuals are classified in three categories: class 0: no dose detected (white); class 1: a positive dose was measured with the lower confidence interval including 0 Gy (hatched); and class 2: a significant positive dose is measured with the lower confidence interval above 0 Gy (black).

La classification des victimes en fonction des doses estimées et pour chaque méthode utilisée, est détaillée dans le tableau I. Les résultats obtenus avec la méthode $50 \mathrm{MS}$ montrent une classification similaire à la méthode de référence pour $50 \%$ des individus (23/46). Les $50 \%$ restant ont été classés avec la méthode $50 \mathrm{MS}$ dans une classe inférieure à celle obtenue par la méthode 250/500 MS. Ces $50 \%$ se décomposent de la façon suivante : 41,3\% classifiés en classe 0 alors qu'ils auraient dû être classifiés en classe $1(19 / 46) ; 4,35 \%$ classifiés en classe 1 alors qu'ils auraient dû l'être en classe $2(2 / 46)$ et enfin 4,35\% classifiés en classe 0 alors qu'ils auraient dû l'être en classe 2 (2/46). 


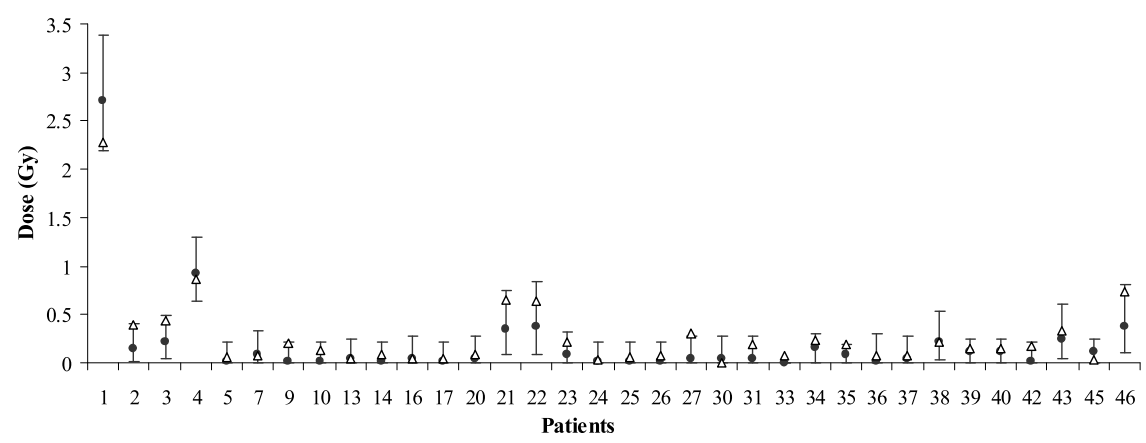

Figure 5 - Doses obtenues pour les individus de la classe 1 (dose positive détectée dont l'intervalle de confiance inférieur inclut la dose 0 Gy) et de la classe 2 (une dose significativement positive est détectée) sont montrés pour la méthode 250/500 MS (losanges noirs) et la méthode ADS (triangles blancs). Les intervalles de confiance sont indiqués uniquement pour les doses obtenues avec la méthode 250/500 MS (losanges noirs).

Results of doses obtained for each individual classified in class 1 (positive dose was measured with the lower confidence interval including $0 \mathrm{~Gy}$ ) and class 2 (significant positive dose is measured with the lower confidence interval above 0 Gy) are showed for the 250/500 MS method (black) and the ADS method (white). The confidence intervals are shown for the reference doses obtained with the 250/500 MS method.

La comparaison des résultats obtenus avec la méthode ADS permet de mettre en évidence une classification similaire à la méthode de référence pour 95,7\% des individus (44/46) (Tab. I). Dans 4,3\% des cas, les individus ont été classés dans une classe inférieure par la méthode ADS à celle obtenue par la méthode 250/500 MS $(2,15 \%$ des individus classifiés en classe 0 alors qu'ils auraient dû être en classe 1 (1/46) et 2,15\% des individus classifiés en classe 1 alors qu'ils auraient dû être en classe 2 (1/46)). Contrairement aux résultats obtenus avec la méthode $50 \mathrm{MS}$, aucun individu de classe 2 n'a été classifié en classe 0 par la méthode ADS.

\subsection{Comparaison de l'efficacité d'estimation de la dose}

Pour les 46 individus, les doses estimées avec la méthode ADS ont toujours été incluses dans l'intervalle de confiance de la dose estimée avec la méthode 250/500 MS et vice versa (Fig. 5). Cependant, un test non-paramétrique de Wilcoxon met en évidence une différence globale significative entre les méthodes MS et $\operatorname{ADS}(Z>1,96 ; p$-value $<0,05)$. En effet, les estimations de dose ont été légèrement plus élevées avec la méthode ADS qu'avec la méthode 250/500 MS.

\subsection{Seuil de sensibilité des différentes méthodes}

Selon la courbe dose-effet obtenue en comptage manuelle (Vaurijoux et al., 2009), le seuil de sensibilité est de 0,53 Gy pour 50 métaphases analysées et 0,16 Gy 


\section{TABLEAU II}

Seuil de sensibilité et temps d'analyse pour les méthodes MS et ADS. Le seuil de sensibilité et son intervalle de confiance sont calculés théoriquement à partir des courbes dose-effet et des courbes d'erreurs standards des deux méthodes (données non présentées). Le temps d'analyse est donné en heures requises par opérateur. Il est à noter que le temps de recherche et d'acquisition par le logiciel Metafer 4 n'est pas inclus.

Sensitivity threshold and time needed for analysis for the MS and ADS methods. Sensitivity threshold and its confidence interval are calculated theoretically with the dose-effect curves and the standard errors curves specific of each methods. The analysis time is shown in hours by operator. The time of metaphase searching and acquisition by the Metafer 4 software isn't included.

\begin{tabular}{cccc}
\hline & Nombre de cellules observées & Seuil de sensibilité & $\begin{array}{c}\text { Temps d'analyse } \\
\text { (heure/operateur) }\end{array}$ \\
\hline \multirow{2}{*}{ MS } & 50 & $0,53 \mathrm{~Gy}[0,002 ; 1,47]$ & 1 \\
& 500 & $0,16 \mathrm{~Gy}[0,01 ; 0,41]$ & 10 \\
\multirow{2}{*}{ ADS } & 500 & $0,44 \mathrm{~Gy}[0,07 ; 0,89]$ & 0,5 \\
& 1000 & $0,31 \mathrm{~Gy}[0,02 ; 0,64]$ & 1 \\
\hline
\end{tabular}

pour 500 métaphases analysées. Le seuil de sensibilité calculé à partir de la courbe dose-effet de la méthode ADS(Vaurijoux et al., 2009) est de 0,31 Gy pour 1000 métaphases analysées (Tab. II). La méthode ADS a donc une meilleure sensibilité pour discriminer les individus réellement exposés que la méthode $50 \mathrm{MS}$. De plus, si l'on augmente le nombre de métaphases analysées à 3000 , le seuil de sensibilité diminue à 0,19 Gy et se rapproche de celui de la méthode 250/500 MS et ceci pour un temps d'analyse divisé par trois (Tab. II).

\section{Discussion}

Cette étude est la première à appliquer la technique de détection automatique des dicentriques pour estimer la dose reçue et réaliser un tri de personnes potentiellement exposées aux rayonnements ionisants. Elle a pour but d'analyser les améliorations que cette méthode automatisée pourrait apporter à la méthode manuelle utilisée actuellement (50 MS puis 250/500 MS) notamment en termes de rapidité et de seuil de sensibilité. Elle a été réalisée à partir d'échantillons collectés dans le cadre d'un accident survenu en 2006 à Dakar dans lequel 63 victimes ont été potentiellement exposées. Pour cet accident, un tri des individus a été fait par comptage des dicentriques sur 50 métaphases puis la dose précise a été estimée par comptage de 250 ou 500 métaphases sur l'ensemble des victimes potentielles. L'étude expérimentale de détection automatique des dicentriques a été faite en utilisant le logiciel d'analyse d'image DCScore (MetaSystems Gmbh) et les différents résultats ainsi obtenus ont été comparés entre eux. 
Le temps nécessaire à l'estimation des doses reçues et la précision de cette estimation sont cruciaux car en cas d'exposition aux rayonnements ionisants, les victimes doivent bénéficier d'une prise en charge médicale rapide et adaptée à leur niveau d'irradiation. Notre étude démontre que dans le cas de l'accident de Dakar, 63 échantillons ont pu être analysés en 5,9 jours par la méthode 50 MS. L'estimation précise des doses par la méthode 250/500 MS a nécessité 15 jours supplémentaires, soit un total de 20,4 jours. L'automatisation de l'analyse (méthode ADS) aurait permis de réaliser les 63 estimations de doses en 8,6 jours pour 1000 métaphases analysées par individu. La méthode ADS est donc plus rapide que la méthode 250/500 MS. Elle reste un peu plus longue que la méthode 50 MS mais permet d'obtenir une précision et une fiabilité de l'estimation du niveau d'exposition beaucoup plus grande. En effet, la comparaison des différentes méthodes montre une sous estimation du niveau d'exposition dans $50 \%$ des cas avec la méthode 50 MS. Cette sous estimation touche en autre 2 des victimes exposées d'une manière significative (classe 2 avec la méthode 250/500 MS) et non détectées par la méthode 50 MS. Par conséquent, le comptage de 50 métaphases parait insuffisamment précis pour un tri pertinent et exhaustif des victimes exposées. En revanche, la méthode ADS permet d'obtenir une concordance des niveaux d'exposition avec la méthode de référence pour $95,7 \%$ des victimes et une sous estimation de classe dans seulement $4,3 \%$ des cas. De plus, cette sous estimation de classe est due essentiellement à l'augmentation de la taille des intervalles de confiance sur la dose estimée avec la méthode ADS. Nous obtenons donc une meilleure estimation des niveaux d'exposition avec la méthode ADS qu'avec la méthode $50 \mathrm{MS}$. Ceci est confirmé lors de l'analyse des doses estimées par chacune des techniques. Ainsi, les doses obtenues par la méthode ADS sont toujours incluses dans l'intervalle de confiance des doses correspondantes mesurées avec la méthode 250/500 MS. Néanmoins la comparaison statistique globale de ces deux méthodes par un test nonparamétrique de Wilcoxon met en évidence une légère une tendance à la surestimation des doses par la méthode ADS.

Dans la littérature, les études sur le tri de population exposée aux rayonnements ionisants à partir d'études biologiques sont rares. Lloyd et al. ont comparé les efficacités de tris basés sur l'analyse de 20 et de 50 métaphases (Lloyd et al., 2000). Ces comparaisons ont été faites à partir de prélèvements sanguins irradiés in vitro simulant l'exposition du corps entier dans une gamme de dose de 0,5 à $8 \mathrm{~Gy}$. À ces niveaux de dose, il a été montré une différence de $10 \%$ et $20 \%$ entre les doses physiques délivrées et les doses estimées après le comptage de 50 et 20 métaphases respectivement. Il est évident que diminuer le nombre de métaphases comptées augmente l'erreur d'estimation de la dose, et ceci surtout pour les gammes de doses faibles comme pour l'accident de Dakar (inférieure à $1 \mathrm{~Gy}$ ). Il est donc important de trouver le bon compromis entre le nombre minimal de métaphases à compter 
pour chaque échantillon (qui conditionne le temps de rendu du résultat de l'estimation de dose) et le seuil de sensibilité minimale acceptable qui dépendra de la situation accidentelle.

L'étude du seuil de sensibilité des différentes méthodes testées démontre que pour 1000 métaphases analysées par la méthode ADS nous obtenons un seuil de détection à $0,31 \mathrm{~Gy}$. Si le nombre de métaphases analysées est porté à 3000 , ce seuil atteint la valeur de 0,19 Gy proche du seuil de sensibilité de la méthode 500 MS. Ceci est d'autant plus intéressant que le temps d'analyse de 3000 métaphases par la méthode ADS reste trois fois plus rapide que celui de 500 métaphases par la méthode MS. La méthode automatisée présente donc un intérêt supplémentaire manifeste puisqu'elle donne la possibilité de moduler son seuil de sensibilité sans entrainer un allongement du temps d'analyse trop important. Cette technique pourrait alors permettre la modulation des paramètres d'analyse en fonction des conditions accidentelles (nombre d'individus exposés).

Un des points d'amélioration de cette approche automatisée concerne l'augmentation de la précision de la mesure et donc la réduction des intervalles de confiance sur la dose. Ceci pourrait être obtenu avec l'augmentation de l'efficacité de détection de la forme des chromosomes et des dicentriques. En effet, avec cette version du logiciel (1.81), environ $75 \%$ des chromosomes sont détectés. De plus, sur la totalité des dicentriques proposés par le système environ $50 \%$ sont validés à $2 \mathrm{~Gy}$. Ceci est cohérent avec les données de la littérature où un laboratoire utilisant le logiciel DCScore (MetaSystems Gmbh) a également mis en évidence une surestimation du nombre de dicentriques principalement aux doses supérieures à 2 Gy (Schunck et al., 2004). C'est pour cette raison que nous avons fait intervenir un opérateur pour vérifier chaque dicentrique détecté par le système. Ce biais n'impacte donc pas la dose néanmoins il rend l'étape de vérification des dicentriques indispensable rallongeant ainsi le temps d'analyse. La modification des critères influant sur la reconnaissance de forme des chromosomes n'est pas possible avec la version actuelle du logiciel DCScore, une nouvelle version plus paramétrable est en cours d'expérimentation.

\section{Conclusion}

Les résultats obtenus avec la détection automatique des dicentriques dans un cas réel d'accident sont particulièrement prometteurs. La méthode ADS est applicable aussi bien dans une situation de tri que dans une estimation précise de la dose. Il est également intéressant de souligner que, lors d'un tri d'un grand nombre de personnes, l'utilisation d'un logiciel d'image commercial permettra d'harmoniser les analyses entre les laboratoires impliqués dans les réseaux de dosimétrie biologique nationaux et internationaux. 
Remerciements. Les auteurs tiennent à remercier Jean-Marc Bertho pour sa relecture attentive et la pertinence de ses commentaires.

\section{RÉFÉRENCES}

Bayley R., Carothers A., Chen X., Farrow S., Gordon J., Ji L., Piper J., Rutovitz D., Stark M., Wald N. (1991) Radiation dosimetry by automatic image analysis of dicentric chromosomes, Mutat. Res. 253, 223-35.

Clairand I., Huet C., Trompier F., Bottollier-Depois J.-F. (2008) Physical dosimetric reconstruction of a radiological accident due to gammagraphy equipment that occurred in Dakar and Abidjan in summer 2006, Radiat. Meas. 43, 698-703.

Edwards A.A., Lloyd D.C., Purrott R.J. (1979) Radiation induced chromosome aberrations and the Poisson distribution, Radiat. Environ. Biophys. 16, 89-100.

Finnon P., Lloyd D. (1992) A preliminary evaluation of the Edinburgh dicentric hunter, J. Radiat. Res. (Tokyo) 33 Suppl, 215-221.

Grégoire E., Gruel G., Martin C., Roch-Lefevre S., Vaurijoux A., Voisin P., Roy L. (2010) Impact des facteurs individuels et environnementaux sur le taux d'aberrations chromosomiques de type translocations - Partie 1 : âge, sexe, tabac, alcool, Radioprotection 45, 153-169.

Lloyd D.C., Edwards A.A., Moquet J.E., Guerrero-Carbajal Y.C. (2000) The role of cytogenetics in early triage of radiation casualties, Appl. Radiat. Isot. 52, 1107-1112.

Papworth D. (1975) Curve fitting by maximum likelihood. Appendix to paper by SAVAGE JRK: Radiation-induced chromosomal aberrations in plant Tradescantia: Dose response curves, Radiat. Botan. 15, 127-131.

Roy L., Roch-Lefevre S., Vaurijoux A., Voisin P., Martin C., Gregoire E., Voisin P. (2007) Optimization of cytogenetic procedures for population triage in case of radiological emergency, Radiat. Meas. 42, 1143-1146.

Roy L., Sorokine-Durm I. and Voisin P. (1996) Comparison between fluorescence in situ hybridization and conventional cytogenetics for dicentric scoring: a first-step validation for the use of FISH in biological dosimetry, Int. J. Radiat. Biol. 70, 665-669.

Schunck C., Johannes T., Varga D., Lorch T., Plesch A. (2004) New developments in automated cytogenetic imaging: unattended scoring of dicentric chromosomes, micronuclei, single cell gel electrophoresis, and fluorescence signals, Cytogenet. Genome Res. 104, 383-389.

Vaurijoux A., Gruel G., Pouzoulet F., Gregoire E., Martin C., Roch-Lefevre S., Voisin P., Voisin P., Roy L. (2009) Strategy for population triage based on dicentric analysis, Radiat. Res. 171, 541-548.

Voisin P., Benderitter M., Claraz M., Chambrette V., Sorokine-Durm I., Delbos M., Durand V., Leroy A., Paillole N. (2001) The cytogenetic dosimetry of recent accidental overexposure, Cell Mol. Biol. 47, 557-564. 